\title{
More Related Gene Pathways to Vincristine-Induced Death Events in a Human T-Acute Lymphoblastic Leukemia Cell Line
}

\author{
Azam Rashidbaghan $^{1}$, Ali Mostafaie*1, Yaghoub Yazdani ${ }^{2}$, Kamran Mansouri $^{3}$
}

\begin{abstract}
Background: Acute lymphoblastic leukemia (ALL) is common in children but rare in adults. Vincristine (VCR) is one of the drugs used at the beginning of treatment. Some genes are resistant to VCR in B-ALL. Methods: Here, we examined the effect of VCR on gene expression changes in a T-ALL cell line, Jurkat. The MTT method was used to determine the $\mathrm{IC}_{50}$ in Jurkat cells treated with different concentrations of VCR for 48 and 72 hours. Total RNA was isolated from the cells and cDNA was prepared. The Human Cancer Drug Target PCR Array kit was used to evaluate the 84 gene expression changes in Jurkat cells. Protein-protein interaction was analyzed by STRING software.

Results: We identified 66 differentially expressed genes as comparison to untreated cells. The response to VCR-induced apoptotic events was remarkable in the pathways of heat shock protein, topoisomerases, protein kinases, cathepsins and cell cycle. In other pathways, there were resistant genes as well as sensitive genes to VCR treatment. Some proteins like HSP90AA1 and ESR1 had determining associations with other proteins.

Conclusions: The results suggest VCR target genes in T-ALL cells may be beneficial biomarkers for ALL treatment and can be used to select appropriate synergistic drugs for VCR.
\end{abstract}

Keywords: ALL, Gene expression profile, Jurkat, PCR array, Vincristine.

\section{Introduction}

ALL is a malignancy of lymphoid cells and is common in children with an incidence of $80 \%$ (1). ALL is rare but fatal in adults (2). New progress for the treatment of ALL has increased survival in children (3). However, the success of these therapies in adults decreases in older ages (2). VCR, corticosteroids and anthracycline are the primary drugs used in chemotherapy of ALL by induction therapy for complete healing of the disease (1). Some patients with ALL (mainly B-ALL) exhibit resistance to anti-leukemic drugs such as VCR (4) and also in vitro resistance to VCR and other drugs has been shown as lethal concentration $\left(\mathrm{LC}_{50}\right)$ in leukemic cells isolated from children diagnosed with B linage ALL (5). The genetic basis of resistance to chemotherapy with prednisolone (PRE), VCR, asparaginase (ASP), or daunorubicin (DNR) was determined in other studies (4, 5). In another study, signal transduction genes related to drug resistance were identified in children with ALL as ex vivo. The drugs included PRE, VCR, ASP, etoposide (VP16), fludarabine (FLU) (6).

VCR is a vinca alkaloid isolated from leaves of Catharanthus roseus (7) and used for the treatment of various cancers (8). VCR influences cells by inhibiting cell division and establishment of microtubules and results in mitotic arrest (9). VCR can induce apoptosis in some leukemic cell lines (7) such as acute Tlymphoblastic CCRF-CEM.f2, pre-B ALL-697 
and Jurkat. Previous studies showed VCR induced apoptosis by extrinsic and intrinsic pathways in Jurkat cells (10). The most studies investigate the gene profile in VCR- resistant BALL cells (4-6). A few studies have explored gene resistant to drugs in T-ALL cells. In a study, gene expression of cells isolated from 7 patients with T-ALL was analyzed. These patients received a four-drug combination of VCR, dexamethasone (DEX), ASP and DNR (3). In another study by Beesley et al. sensitive and resistance pathways to VCR and other antileukemia drugs were determined using microarray data from T-ALL cell lines derived from pediatric ALL bone marrow specimens. A gene model was made based on resistant genes and used for 3 patients in primary cohort. The patients were treated with 10 drugs and. The evaluation was indicated that T-ALL patients to be sensitive to 6-mercaptopurine. The aim of this study was finding the genes and pathways associated with resistance (11). However, there is not a study that especially evaluates gene expression of pathways associated with cancer drug targets in T-ALL cell line or patients treated with VCR. The aim of this study was to determine a gene expression profile of the TALL cell line in response to VCR treatment. So, we evaluated gene expression changes of human cancer drug targets in the T-ALL Jurkat cells to identifying relevant pathways to VCR treatment.

\section{Materials and Methods Cell culture}

The human T-lymphoblastic leukemia cell line (Jurkat, cell line number: C121) was purchased from the cell bank of the Pasture Institute (Tehran, Iran). The cell line identified in DNA Typing laboratory of the Pasture Institute (by STR analysis method). Also, the cells were tested for microbial and fungal contaminations, especially mycoplasma contamination, in Microbial Quality Control laboratory of the Pasture Institute. Cells cultured in RPMI-1640 medium (Gibco, USA, 11875093 ) with supplemented with $10 \%$ heatinactivated fetal bovine serum (FBS) (Gibco, USA, A25904DG) and penicillin- streptomycin (Gibco, USA, 15140122) and incubated at $37{ }^{\circ} \mathrm{C}$ in a $5 \% \mathrm{CO} 2$ atmosphere with $95 \%$ humidity. When the cells were obtained from cell bank, first, were cultured and passaged for one month. After that, the cells were harvested in tubes containing DMSO and frizzed and maintained in the nitrogen tank. These cells were cultured and passaged again to perform experiments after about one year.

\section{MTT assay}

Cell growth inhibition was measured based on the 3-(4, 5-dimethylthiazol-2-yl)-2, 5diphenyl-tetrazoliumbromide (MTT) assay. $6 \times 10^{4}$ cells per well of Jurkat were seeded into a 96-well plate and treated with different concentrations of VCR $(0.05,0.1,0.5$ and 1 $\mu \mathrm{g} / \mathrm{ml})$. After 48- and 72-hours incubation at $37^{\circ} \mathrm{C}, 20 \mu \mathrm{l}$ of MTT solution $(5 \mathrm{mg} / \mathrm{ml}$ in PBS) (Sigma, USA, A101161) was added into each well and cells were incubated at $37^{\circ} \mathrm{C}$ for 4 hours. After adding DMSO to wells to dissolve the formazan crystals, plates were read using a plate reader at $570 \mathrm{~nm}$ against $630 \mathrm{~nm}$. The experiment was performed three times. The $50 \%$ inhibitory concentration (IC50) was determined. Cell growth (\%) was calculated as following (12):

Cell growth $(\%)=\frac{A_{490 \mathrm{~nm}}(\text { sample })}{A_{490 \mathrm{~nm}}(\text { control })} \times 100$

\section{Isolation of total RNA and cDNA preparation}

Jurkat cells $(3.5 \times 105$ cells $/ \mathrm{ml})$ were treated with vincristine (in a concentration based on IC50) for $72 \mathrm{~h}$. Total RNA was extracted from the harvested cells using RNX-Plus (Sinaclon, Tehran, Iran, RN7713C). The quality and quantity of RNA were estimated by the Picodrop machine (Picodrop Microliter, USA). Then, RNA treated with RNase- free DNase I, for removing genomic DNA, was converted to cDNA using First Strand cDNA Synthesis kit (Parstous, Iran, A101161; Thermo Fisher Scientific, USA, EN0521) according to manufacturer's instruction. 


\section{Gene expression analysis by PCR array}

The Human cancer drug target $\mathrm{RT}^{2}$ Profiler ${ }^{\mathrm{TM}}$ PCR Array (Qiagen, USA, PAHS-507Z) was used to screen 84 gene expression changes of 17 pathways. Real-time PCR was performed according to the manufacturer's instruction. The diluted cDNA was mixed with RT2 SYBR Green/ROX PCR master mix (Qiagen, USA, PAHS-507Z) and RNase-free water with a final volume of $2700 \mu 1$. Subsequently, $25 \mu 1$ of the reaction mixture was placed in to each well of a 96 well plate and qRT-PCR was carried out on ABI 7300 machine (Applied Biosystems, USA) by a three -step cycling program including one cycle in $95^{\circ} \mathrm{C}$ for 10 min, 40 cycles in $95^{\circ} \mathrm{C}$ for $15 \mathrm{~s}$ and, finally, 60 ${ }^{\circ} \mathrm{C}$ for $1 \mathrm{~min}$. Each experiment was carried out in duplicate $(\mathrm{n}=2)$. The $\Delta \Delta \mathrm{Ct}$ method was used for PCR array data analysis. The analysis was performed using the software in the web portal of Qiagene company (http://pcrdataanalysis.sabiosciences.com /pcr/arrayanalysis.php) The data normalized with five housekeeping genes including $B 2 M$, HPRT1, RPL13A, GAPDH, ACTB.

\section{Protein-interaction analysis}

We model a protein interaction network to analyze the association between human cancer drug target genes induced with VCR in Jurkat cells. STRING 11.0 software was used to construct protein-protein interaction map of genes.

\section{Statistical analysis}

The data are presented as mean \pm standard error mean (SEM). Graph of MTT assay test was processed with GraphPad prism 6.0.

\section{Results}

\section{Cell growth assay}

The proliferation percent of Jurkat cells treated with different doses of VCR was examined during 48 and $72 \mathrm{~h}$ using MTS assay. Table 1 shows $\mathrm{IC}_{50}$ values VCR in Jurkat cells.

Table 1. $\mathrm{IC}_{50}$ of VCR in Jurkat cells treated for 48 and $72 \mathrm{~h}$.

\begin{tabular}{cc}
\hline Time & $\mathbf{I C}_{\mathbf{5 0}}(\boldsymbol{\mu g} / \mathbf{m l}) \mathbf{9 5 \%}$ Confidence Interval \\
\hline $48 \mathrm{~h}$ & $0.54(0.2493$ to 1.176$)$ \\
$72 \mathrm{~h}$ & $0.17(0.1086$ to 0.2640$)$ \\
\hline
\end{tabular}

\section{Gene expression changes}

PCR array analysis was performed to identify molecular targets that are affected by VCR in Jurkat cells. Human cancer drug target array results showed that 66 genes of 84 genes had expression levels higher or less than 1.5 in Jurkat cells treated with VCR $(0.5 \mu \mathrm{g} / \mathrm{ml})$ in comparison to untreated cells, 50 genes were down-regulated, and 16 genes were upregulated (Table 2). In the scatter plot, the central line indicates unchanged gene expression, blue and yellow dots were downregulated and up-regulated genes, respectively (Fig. 1).

Table 2. The results of the PCR array for Jurkat cells treated with VCR. Values less and more than 1.5 show gene downregulation and up-regulation as compared to non-treated cells (control group).

\begin{tabular}{llll}
\hline UniGene ID & Gene Description & $\begin{array}{l}\text { Gene } \\
\text { symbol }\end{array}$ & $\begin{array}{l}\text { Fold } \\
\text { regula } \\
\text { tion }\end{array}$ \\
\hline Apoptosis & & & \\
\hline Hs.150749 & B-cell CLL/lymphoma 2 & BCL2 & - \\
\hline Hs.744872 & Baculoviral IAP repeat-containing 5 & BIRC5 & 1.95 \\
\hline PI-3 Kinases \& Phosphatases & & \\
\hline Hs.338207 & Mechanistic target of rapamycin (serine/threonine kinase) & MTOR & 2.49 \\
\hline Hs.736397 & Phosphoinositide-3-kinase, class 2, alpha polypeptide & PIK3C2A & -2.78 \\
\hline
\end{tabular}




\begin{tabular}{|c|c|c|c|}
\hline Hs.656958 & Phosphoinositide-3-kinase, class 3 & PIK3C3 & -5.35 \\
\hline Hs.553498 & Phosphoinositide-3-kinase, catalytic, alpha polypeptide & PIK3CA & -9.09 \\
\hline \multicolumn{4}{|c|}{ Growth Factors \& Receptors } \\
\hline Hs.488293 & Epidermal growth factor receptor & EGFR & -1.77 \\
\hline Hs.446352 & $\begin{array}{l}\text { V-erb-b2 erythroblastic leukemia viral oncogene homolog } \\
2, \text { neuro/glioblastoma derived oncogene homolog (avian) }\end{array}$ & ERBB2 & 1.90 \\
\hline Hs.118681 & $\begin{array}{l}\text { V-erb-b2 erythroblastic leukemia viral oncogene homolog } \\
3 \text { (avian) }\end{array}$ & ERBB3 & -4.25 \\
\hline Hs.390729 & $\begin{array}{l}\text { V-erb-a erythroblastic leukemia viral oncogene homolog } 4 \\
\text { (avian) }\end{array}$ & ERBB4 & -2.13 \\
\hline Hs.11392 & $\begin{array}{l}\text { C-fos induced growth factor (vascular endothelial growth } \\
\text { factor D) }\end{array}$ & FIGF & -3.43 \\
\hline Hs.594454 & $\begin{array}{l}\text { Fms-related tyrosine kinase } 1 \text { (vascular endothelial growth } \\
\text { factor/vascular permeability factor receptor) }\end{array}$ & FLT1 & -4.92 \\
\hline Hs.646917 & Fms-related tyrosine kinase 4 & FLT4 & 2.45 \\
\hline Hs. 160562 & Insulin-like growth factor 1 (somatomedin C) & $1 \mathrm{GF} 1$ & 1.57 \\
\hline Hs. 643120 & Insulin-like growth factor 1 receptor & IGF1R & -2.48 \\
\hline Hs.700350 & Insulin-like growth factor 2 (somatomedin A) & IGF2 & 2.91 \\
\hline Hs.479756 & $\begin{array}{l}\text { Kinase insert domain receptor (a type III receptor tyrosine } \\
\text { kinase) }\end{array}$ & KDR & -4.00 \\
\hline Hs.479754 & $\begin{array}{l}\text { V-kit Hardy-Zuckerman } 4 \text { feline sarcoma viral oncogene } \\
\text { homolog }\end{array}$ & KIT & -5.17 \\
\hline Hs.74615 & Platelet-derived growth factor receptor, alpha polypeptide & PDGFRA & - \\
\hline Hs.509067 & Platelet-derived growth factor receptor, beta polypeptide & PDGFRB & - \\
\hline \multicolumn{4}{|c|}{ Drug Metabolism } \\
\hline Hs.391464 & $\begin{array}{l}\text { ATP-binding cassette, sub-family C (CFTR/MRP), } \\
\text { member } 1\end{array}$ & $\mathrm{ABCC} 1$ & -1.66 \\
\hline Hs. 523836 & Glutathione S-transferase pi 1 & GSTP1 & -1.88 \\
\hline Hs. 196384 & $\begin{array}{l}\text { Prostaglandin-endoperoxide synthase } 2 \text { (prostaglandin G/H } \\
\text { synthase and cyclooxygenase) }\end{array}$ & PTGS2 & - \\
\hline Hs.435136 & Thioredoxin & TXN & - \\
\hline Hs.690011 & Thioredoxin reductase 1 & TXNRD1 & -5.95 \\
\hline \multicolumn{4}{|c|}{ G Protein Signaling } \\
\hline Hs. 247077 & Ras homolog gene family, member A & RHOA & -2.13 \\
\hline Hs.502876 & Ras homolog gene family, member B & RHOB & 8.06 \\
\hline \multicolumn{4}{|c|}{ Hormone Receptors } \\
\hline Hs.744830 & Estrogen receptor 1 & ESR1 & 2.09 \\
\hline Hs.660607 & Estrogen receptor 2 (ER beta) & ESR2 & - \\
\hline Hs.32405 & Progesterone receptor & PGR & -10.68 \\
\hline \multicolumn{4}{|c|}{ Heat Shock Proteins } \\
\hline Hs. 525600 & $\begin{array}{l}\text { Heat shock protein } 90 \mathrm{kDa} \text { alpha (cytosolic), class A } \\
\text { member } 1\end{array}$ & $\begin{array}{l}\text { HSP90AA } \\
1\end{array}$ & -2.69 \\
\hline Hs.192374 & Heat shock protein 90kDa beta (Grp94), member 1 & HSP90B1 & -2.95 \\
\hline \multicolumn{4}{|c|}{ Receptor Tyrosine Kinase Signaling } \\
\hline Hs. 525622 & V-akt murine thymoma viral oncogene homolog 1 & AKT1 & - \\
\hline Hs.631535 & V-akt murine thymoma viral oncogene homolog 2 & AKT2 & - \\
\hline Hs.444356 & Growth factor receptor-bound protein 2 & GRB2 & -4.68 \\
\hline
\end{tabular}




\begin{tabular}{|c|c|c|c|}
\hline \multicolumn{4}{|l|}{ Cathepsins } \\
\hline Hs.520898 & Cathepsin B & CTSB & - \\
\hline Hs. 654447 & Cathepsin D & CTSD & -1.88 \\
\hline Hs.731507 & Cathepsin L1 & CTSL & -3.51 \\
\hline Hs. 181301 & Cathepsin S & CTSS & -4.75 \\
\hline \multicolumn{4}{|l|}{ Cell cycle } \\
\hline Hs.732435 & Cyclin-dependent kinase 1 & CDK1 & -2.49 \\
\hline Hs.19192 & Cyclin-dependent kinase 2 & CDK2 & -1.77 \\
\hline Hs.95577 & Cyclin-dependent kinase 4 & CDK4 & - \\
\hline Hs. 647078 & Cyclin-dependent kinase 5 & CDK5 & -1.90 \\
\hline Hs. 184298 & Cyclin-dependent kinase 7 & CDK7 & - \\
\hline Hs.382306 & Cyclin-dependent kinase 8 & CDK8 & -6.60 \\
\hline Hs. 150423 & Cyclin-dependent kinase 9 & CDK9 & -3.18 \\
\hline Hs.437705 & Cell division cycle 25 homolog A (S. pombe) & CDC25A & - \\
\hline Hs.484551 & Mdm2 p53 binding protein homolog (mouse) & MDM2 & -7.60 \\
\hline Hs.715871 & Mdm4 p53 binding protein homolog (mouse) & MDM4 & -10.43 \\
\hline Hs.492203 & Telomerase reverse transcriptase & TERT & 1.81 \\
\hline \multicolumn{4}{|c|}{ Topoisomerases, Type II } \\
\hline Hs. 156346 & Topoisomerase (DNA) II alpha 170kDa & TOP2A & -3.56 \\
\hline Hs.475733 & Topoisomerase (DNA) II beta $180 \mathrm{kDa}$ & TOP2B & -5.96 \\
\hline \multicolumn{4}{|c|}{ Transcription Factors } \\
\hline Hs.631535 & V-akt murine thymoma viral oncogene homolog 2 & ATF2 & -7.34 \\
\hline Hs.597216 & $\begin{array}{l}\text { Hypoxia inducible factor } 1 \text {, alpha subunit (basic helix- } \\
\text { loop-helix transcription factor) }\end{array}$ & HIF1A & -8.01 \\
\hline Hs.521181 & Interferon regulatory factor 5 & IRF5 & -2.37 \\
\hline Hs.618430 & $\begin{array}{l}\text { Nuclear factor of kappa light polypeptide gene enhancer in } \\
\text { B-cells } 1\end{array}$ & NFKB1 & - \\
\hline Hs.437460 & Tumor protein p53 & TP53 & - \\
\hline \multicolumn{4}{|c|}{ Protein Kinases } \\
\hline Hs. 250822 & Aurora kinase A & AURKA & -6.81 \\
\hline Hs.442658 & Aurora kinase B & AURKB & -3.03 \\
\hline Hs.98338 & Aurora kinase $\mathrm{C}$ & AURKC & -3.60 \\
\hline Hs.592049 & Polo-like kinase 1 & PLK1 & -1.56 \\
\hline Hs.398157 & Polo-like kinase 2 & PLK2 & -8.26 \\
\hline Hs. 632415 & Polo-like kinase 3 & PLK3 & -1.96 \\
\hline Hs.172052 & Polo-like kinase 4 & PLK4 & -3.51 \\
\hline Hs.531704 & Protein kinase $\mathrm{C}$, alpha & PRKCA & 1.75 \\
\hline Hs.460355 & Protein kinase $\mathrm{C}$, beta & PRKCB & 1.75 \\
\hline Hs. 155342 & Protein kinase $\mathrm{C}$, delta & PRKCD & -2.01 \\
\hline Hs.580351 & Protein kinase $\mathrm{C}$, epsilon & PRKCE & -3.35 \\
\hline \multicolumn{4}{|c|}{ RAS Signaling } \\
\hline Hs.37003 & V-Ha-ras Harvey rat sarcoma viral oncogene homolog & HRAS & - \\
\hline Hs.505033 & V-Ki-ras2 Kirsten rat sarcoma viral oncogene homolog & KRAS & -2.18 \\
\hline
\end{tabular}


Gene Profile of VCR Treated T-ALL Cells

\begin{tabular}{lllll}
\hline Hs.486502 & Neuroblastoma RAS viral (v-ras) oncogene homolog & NRAS & \multicolumn{1}{l}{-3.05} \\
\hline Histone Deacetylases & & \\
\hline Hs.88556 & Histone deacetylase 1 & HDAC1 & - \\
\hline Hs.744132 & Histone deacetylase 11 & HDAC11 & - \\
\hline Hs.3352 & Histone deacetylase 2 & HDAC2 & -9.53 \\
\hline Hs.519632 & Histone deacetylase 3 & HDAC3 & -2.23 \\
\hline Hs.20516 & Histone deacetylase 4 & HDAC4 & 1.71 \\
\hline Hs.6764 & Histone deacetylase 6 & HDAC6 & - \\
\hline Hs.200063 & Histone deacetylase 7 & HDAC7 & - \\
\hline Hs.310536 & Histone deacetylase 8 & HDAC8 & 1.62 \\
\hline Poly ADP-Ribose Polymerases & & \\
\hline Hs.177766 & Poly (ADP-ribose) polymerase 1 & PARP1 & -1.50 \\
\hline Hs.409412 & Poly (ADP-ribose) polymerase 2 & PARP2 & 1.50 \\
\hline Hs.744855 & Poly (ADP-ribose) polymerase family, member 4 & PARP4 & -8.14 \\
\hline Hs.370267 & Tankyrase, TRF1-interacting ankyrin-related ADP-ribose & TNKS & -2.67 \\
\hline Structural protein & & \\
\hline Hs.158336 & Netrin 3 & NTN3 & 12.35 \\
\hline
\end{tabular}

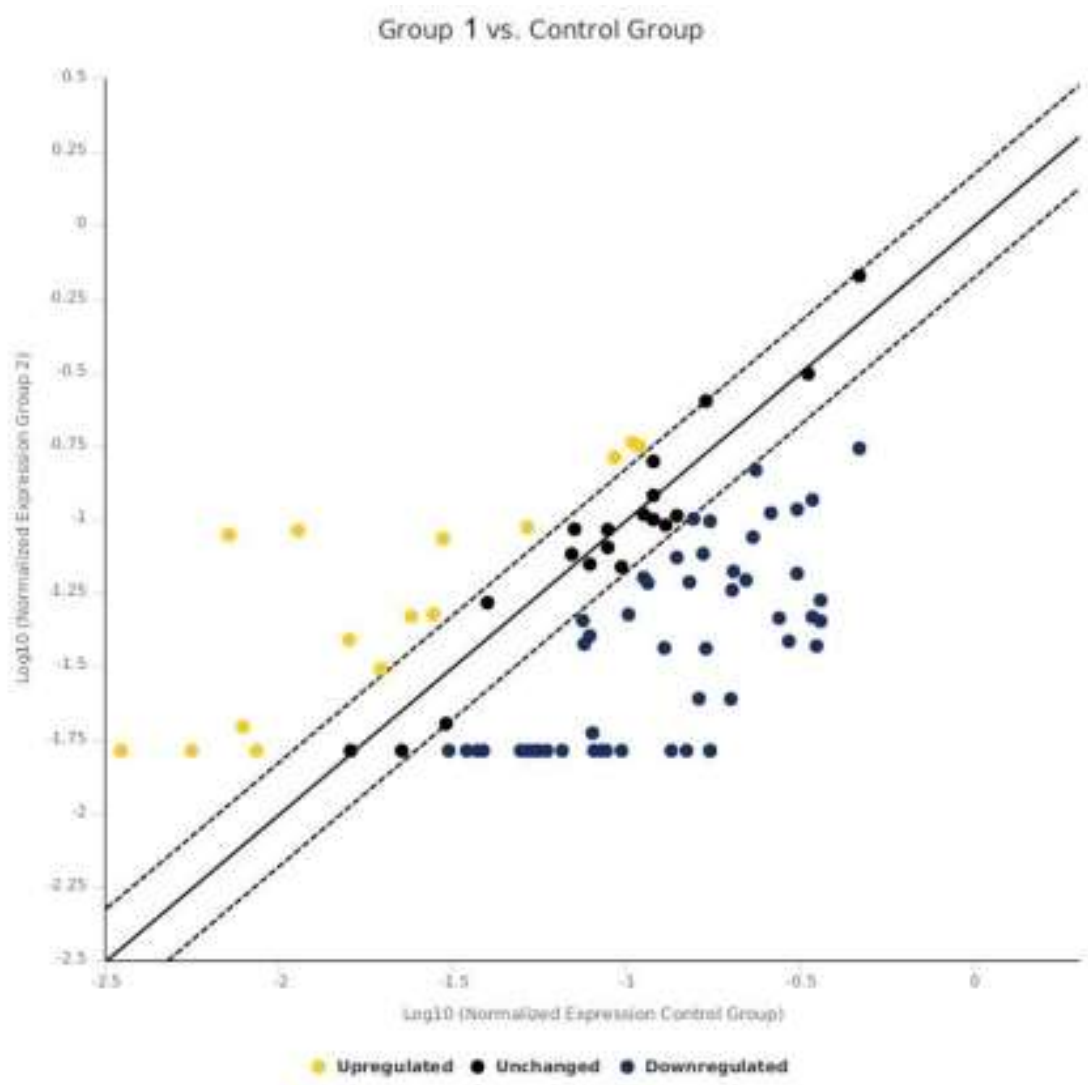

Fig. 1. Effect of VCR on gene expression profiles of Jurkat cells. Eighty-four human cancer drug target genes were evaluated in Jurkat cells treated by VCR in a concentration of $0.5 \mu \mathrm{g} / \mathrm{ml}$. Scatter plot diagram compares the gene expression changes of cells treated by VCR to control cells (untreated cells). The yellow and blue dots show up-regulated and down- regulated genes, respectively and the black dots are un-changed genes. 


\section{Functional and physical interaction network} for VCR-induced genes in Jurkat cells

To identify associations between differentially expressed genes in Jurkat cells treated with VCR, a protein interaction network was made using information of the STRING database. In this network, proteins (nods) joint to each other by lines functionally and physically. Totally, 244 putative interactions among 66 proteins were observed that are production of up- and down- regulated genes (Fig. 2). Among these activated proteins, HSP90AA1 had the most interactions with other proteins of modules. Also, CDK1, PRKCA, RHOA, GRB2, PIK3CA, ERBB2 and ERBB4 interacted with many proteins. Proteins of pathways including drug metabolism, cathepsins and poly ADPribose polymerases had the fewer interactions with other pathways.

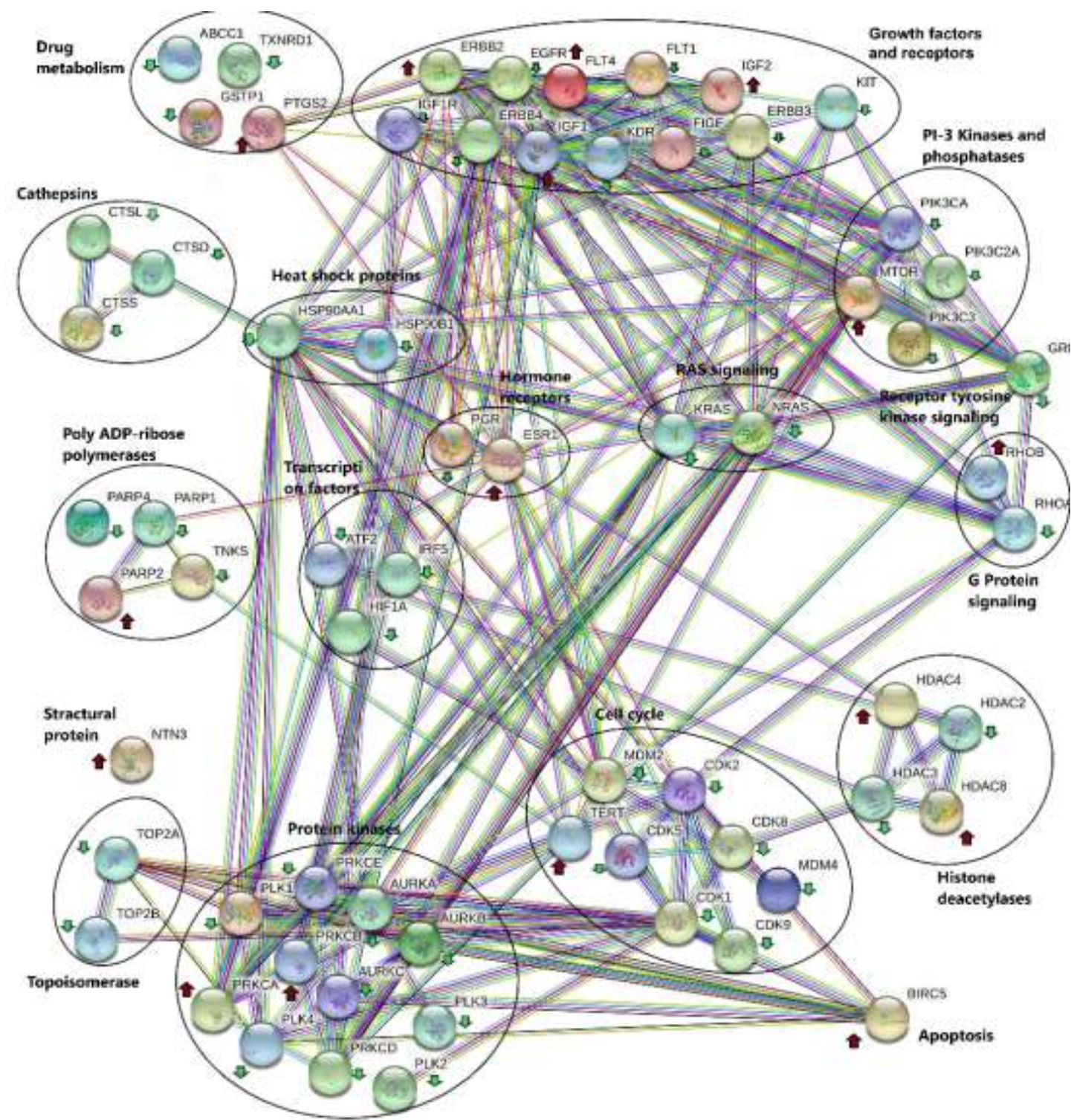

Fig. 2. The cancer functional and physical interaction network among genes induced by VCR in Jurkat cells. Nods and lines represent proteins and protein-protein associations, respectively. The proteins were manually put in clusters based on gene pathways of human cancer drug target PCR array kit. The name of each cluster was labeled above it. Overexpressed and lowexpressed proteins are marked with dark red and green arrows, respectively. The network includes 66 proteins and 244 interactions among them. Green, red, blue, black, light blue, brown and purple lines indicate neighborhood, gene fusion, cooccurrence, co-expression, experiments, database, text-mining, and homology, respectively. STRING 11.0 was used to construct the interaction network. 


\section{Discussion}

The literature review demonstrates the reports about gene pathways and mechanism of resistance to VCR in ALL cells of patients. However, most of them are B-lineage cells or a combination of B- and T- cells (4-6). Then, the effect of VCR on gene profile of T-ALL cells has not been specifically evaluated until now. Here, we investigated human cancer drug target gene expression changes in Jurkat cells treated by VCR. The mRNA level of 84 genes in seventeen pathways was compared between treated cells and untreated cells (negative control). The pathways included apoptosis, PI3-kinase and phosphatase, growth factors and receptors, drug metabolism, $G$ protein signaling, hormone receptors, heat shock proteins, receptor tyrosine kinase signaling, cathepsins, cell cycle, topoisomerase type II, transcription factors, protein kinases, RAS signaling, histone deacetylases, poly ADPribose polymerase, structural protein. The gene expression consideration revealed that VCR induced cell cycle inhibition by decreasing mRNA levels of CDK1, CDK2, CDK5, CDK8 and CDK9. Cyclin-dependent kinases (CDKs) are serine-threonine protein kinases with a direct role in the regulation of the mitosis cell cycle and an indirect role in meiosis, transcription, and DNA repair (13). VCR treatment, also, caused down-regulation of MDM2 and MDM4. MDM2/MDM4 complex decreases the protein level of p53 (a tumor suppressor) (14). TERT was another gene in the cell cycle pathway and was up regulated in this study. TERT is a part of telomerase that catalyzes increasing and stabilizing telomere length. The activity of telomerase results in immortalizing the cells and is critical in carcinogenesis. Previous studies showed telomerase activity was higher in children with acute leukemia in comparison to healthy persons (15). So TERT was one of evaluated genes that resisted against cytotoxic effect of VCR in Jurkat cells.

The expression of CTSD, CTSL and CTSS was decreased under the influence of VCR. The intensity of expression reduction was higher for CTSL and CTSS genes. Cathepsins (CTS) play an important role in tumor progression. Overexpression of these proteins is correlated with cancers (16).

Apoptosis effects of VCR on Jurkat cells led to down-regulating TOP2A, TOP2B, HSP90AA1, HSP90B1 and transcription factors genes (ATF2, HIF1A). Topoisomerases are necessary for many DNA functions during normal cell growth and, also, are trigger in various physiologic and nonphysiologic stresses because of their role in DNA breaking and reconnecting (17). ATF2, HIF1 and HSP90 are anti-apoptotic proteins and overexpressed in cell stress conditions (18-20). As shown in Fig. 2, both of TOP2A and TOP2B have direct interaction with CDK1. HSP90AA1 is a key protein in the gene network activated by VCR and has the most association with other proteins.

The expression level of HDAC2 and HDAC3 in treated cells was 9.53 and 2.23 times lower than untreated cells, respectively. HDAC4 and HDAC8 were up regulated, but they can reduce apoptotic effect of VCR because in their expression level changes are very minor. Histone deacetylases (HDACs) result in chromatin condensation by histone deacetylation and facilitate gene expression. Several studies have shown the anti-leukemic activity of HDAC inhibitors and the potential role of HDACs in treatment of leukemia (21). It was indicated VCR decreased gene transcription in ALL cells, so that the association between HDAC3 and ATF2 (a transcription factor) was shown in Fig. 2.

Down-regulation of the PI3- kinase and phosphatase genes (PIK3C2A, PIK3C3 and PIK3CA) and growth factors and receptor genes (EGFR, ERBB3, ERBB4, FLT1, IGF1R, KDR and KIT) was a response to apoptosis signals of VCR. The member of PIK3C group play several roles in angiogenesis, cell growth and tumor formation (through activity in mTOR signaling pathway) and cell cycle $(22,23)$. PIK3CA had an interaction with MDM2 in Fig. 2. Bartram et al 
described that IGF1R inhibition was effective in decreasing proliferation of T-ALL cells (Jurkat and Molt-4) resistant to VCR and ASP (24). Our results confirmed IGF1R, as a growth receptor, was one of the target genes in Jurkat cells treated by VCR. KIT is a tyrosine kinase receptor and leukemia is the most cancer related to KIT gene mutation (25).

Overexpression of glutathione-s-transferase p1 (GSTP1) is related to resistance to anticancer drugs (26). Expression of ABCC1, also, increases in many human cancer cell lines with multi-drug resistance (27). Then VCR increased the sensitivity of Jurkat cells to drug effect by down-regulating GSTP1 and ABCC 1 genes.

According to our results, signaling pathways and hormone receptors were affected in T-ALL cells by VCR treatment. So that, RHOA (G protein signaling), GRB2 (receptor tyrosine kinase signaling) and PGR (a hormone receptor) were downregulated. All the genes in protein kinases pathway were activated by VCR induction that the most of them enhanced cell death. AURKA, AURKB and AURKC are three members of serine/threonine kinase family and regulators of mitosis and meiosis. Overexpression of AURKA and AURKB was detected in solid tumors and ALL and acute myeloid leukemia (AML) (28). In this research, the expression level of PLK genes was decreased in Jurkat cells. PLK1 overexpressed in various cancers and high expression of PLK4 is related to many cases of metastatic cancers. PLK2 is a tumor suppressor but PLK3 has a low expression in many neoplastic tissues, and it seems PLK3 is a pro-apoptotic protein (29). High expression of PRKC was shown in a study by Lei et al. They demonstrated overexpression of Rack1 (a receptor of PKC) resulted in raising expression level of $\mathrm{PKC} \alpha$ in resistant T-ALL cell lines (Jurkat and CCRFCEM) to VCR (30). Down- regulation of

\section{References}

1. Terwilliger T, Abdul-Hay M. Acute lymphoblastic leukemia: a comprehensive review
PRKCD and PRKCE genes had a positive effect on Jurkat cell apoptotic events in our study.

Decreased mRNA level of KRAS, PARP1 and TNKS genes were other feedbacks of apoptotic events in Jurkat cells due to treatment by VCR.

Some evaluated genes showed resistance to VCR apoptosis induction by increasing expression level, including BIRC5, MTOR, ERBB2, FLT4, IGF1, IGF2, ESR1, NRAS, PARP2. But also, decreasing mRNA level of some genes was resulted in cell survival and resistance to cell death, including TXNRD1, IRF5 and PARP4. From up-regulated genes, ESR1 is the most important resistant gene and interacts with four up-regulated genes of other pathways including TERT, HDAC4, MTOR and IGF1 which had a negative effect on VCR apoptotic process (Fig. 2). ESR1 stimulates the proliferation of cancer cells (31).

In conclusions, in most of evaluated pathways, there were genes with expression changes resulted from VCR apoptotic signals, although some genes were without changes and some genes were resistant to VCR treatment. In this study exactly VCR target genes in T-ALL cells were determined that can be used as biomarkers for ALL therapy and future studies about new anti-leukemic agents. Also, these results can be used to select appropriate drugs to synergize the effect of VCR to improve ALL treatment.

\section{Acknowledgements}

The authors thank Mr. Shahram Parvaneh (Medical Biology Research Center, Kermanshah University of Medical Sciences, Kermanshah, Iran) and Dr. Saeed Mohammadi (School of Advanced Technology, Golestan University of Medical Sciences, Gorgan, Iran) for their valuable advice and technical support. The authors declare that there is no conflict of interest.

and 2017 update. Blood Cancer J. 2017;7(6): e577. 
2. Pulte D, Jansen L, Gondos A, Katalinic A, Barnes B, Ressing M, et al. Survival of adults with acute lymphoblastic leukemia in Germany and the United States. PLOS ONE. 2014;9(1):e85554. 3. Samuels AL, Beesley AH, Yadav BD, Papa RA, Sutton R, Anderson D, et al. A pre-clinical model of resistance to induction therapy in pediatric acute lymphoblastic leukemia. Blood Cancer J. 2014;4(8): e232.

4. Holleman A, Cheok MH, Boer MLD, Yang W, Veerman AJP, Kazemier KM, et al. Geneexpression patterns in drug-resistant acute lymphoblastic leukemia cells and response to treatment. N Engl J Med. 2004;351(6):533-42.

5. Lugthart $\mathrm{S}$, Cheok MH, Den Boer ML, Yang W, Holleman A, Cheng C, et al. Identification of genes associated with chemo-therapy crossresistance and treatment response in childhood acute lymphoblastic leukemia. Cancer Cell. 2005;7(4):375-86.

6. Szczepanek J, Pogorzala M, Jarzab M, Oczko-Wojciechowska M, Kowalska M, Tretyn A. Expression profiles of signal transduction genes in ex vivo drug-resistant pediatric acute lymphoblastic leukemia. Anticancer Res. 2012;32(2):503-6.

7. Groninger $\mathrm{E}$, Meeuwsen-De Boar $\mathrm{T}$, Koopmans P, Uges D, Sluiter W, Veerman A, et al. Pharmacokinetics of vincristine monotherapy in childhood acute lymphoblastic leukemia. Pediatr Res. 2002;52(1):113-8.

8. Gonzalez-Cid M, Cuello MT, Larripa I. Comparison of the aneugenic effect of vinorelbine and vincristine in cultured human lymphocytes. Mutagenesis 1999;14(1):63-66.

9. Ceppi F, Langlois-Pelletier C, Gagné V, Rousseau J, Ciolino C, De Lorenzo S, et al. Polymorphisms of the vincristine pathway and response to treatment in children with childhood acute lymphoblastic leukemia. Pharmacogenomics. 2014;15(8):1105-1116.

10. Groninger E, Meeuwsen-De Boer GJ, De Graaf SSN, Kamps WA, De Bont ESJM. Vincristine induced apoptosis in acute lymphoblastic leukemia cells: a mitochondrial controlled pathway regulated by reactive oxygen species?. Int J Oncol. 2002;21(6):1339-45.

11. Beesley AH, Firth MJ, Anderson D, Samuels AL, Ford J, Kees UR. Drug-Gene Modeling in
Pediatric T-Cell Acute Lymphoblastic Leukemia Highlights Importance of 6-Mercaptopurine for Outcome. Cancer Res. 2013;73(9):2749-59.

12. Looi CY, Arya A, Cheah FK, Muharram B, Leong $\mathrm{KH}$, Mohamad $\mathrm{K}$, et al. Induction of apoptosis in human breast cancer cells via caspase pathway by vernodalin isolated from Centratherum anthelminticum (L.) seeds. PLOS ONE. 2013;8(2):e56643.

13. Aleem E, Arceci RJ. Targeting cell cycle regulators in hematologic malignancies. Front Cell Dev Biol. 2015;3:16.

14. Mancini F, Teveroni E, Conza GD, Monteleone V, Arisi I, Pellegrino M, et al. MDM4 actively restrains cytoplasmic mTORC1 by sensing nutrient availability. Mol Cancer. 2017;16(1):55.

15. Sheng X, Tong N, Tao G, Luo D, Wang M, Fang Y, et al. TERT polymorphisms modify the risk of acute lymphoblastic leukemia in Chinese children. Carcinogenesis. 2013;34(1):228-235.

16. Turk V, Stoka V, Vasiljeva O, Renko M, Sun $\mathrm{T}$, Turk B, et al. Cysteine cathepsins: from structure, function and regulation to new frontiers. Biochimica et Biophysica Acta (BBA) - Proteins and Proteomics. 2012;1824(1):68-88.

17. Li TK, Liu LF. Tumor cell death induced by topoisomerase-targeting druges. Annual Review of Pharmacology and Toxicology. 2001; 41:5377.

18. Redell MS, Tweardy DJ. Targeting transcription factors in cancer: Challenges and evolving strategies. Drug Discov Today Technol. 2006;3(3):261-7.

19. Lopez-Bergami P, Lau E, Ronai Z. Emerging roles of ATF2 and the dynamic AP1 network in cancer. Nature Reviews Cancer. 2010;10(1):6576.

20. Thomas X, Campos L, Le QH, Guyotat D. Heat shock proteins and acute leukemias. Hematology. 2005;10(3):225-35.

21. Verza FA, Das U, AL, Dimmock JR, Marins $\mathrm{M}$. Roles of histone deacetylases and inhibitors in anticancer therapy. Cancers (Basel). 2020;12(6): 1664 .

22. Laplante M, Sabatini DM. mTOR signaling at a glance. J Cell Sci. 2009;122(Pt 20):3589-94.

23. Liang $X$, Xin X, Qi D, Fu C, Ding $M$. Silencing the PIK3CA gene enhances the 
sensitivity of childhood leukemia cells to chemotherapy drugs by suppressing the phosphorylation of Akt. Yonsei Med J. 2019;60(2):182-190.

24. Bartram I, Erben U, Ortiz-Tanchez J, Blunert K, Schlee C, Neumann M, et al. Inhibition of IGF1-R overcomes IGFBP7-induced chemotherapy resistance in T-ALL. BMC Cancer. 2015;15:663.

25. Abbaspour Babaei M, Kamalidehghan B, Saleem M, Huri HZ, Ahmadipour F. Receptor tyrosine kinase (c-Kit) inhibitors: a potential therapeutic target in cancer cells. Drug Des Devel Ther. 2016;10:2443-59.

26. Hameda NAM, Ghallaba O, El-Neilyb D. Glutathione-S-transferase P1 as a risk factor for Egyptian patients with chronic myeloid leukemia. The Egyptian Journal of Haematology. 2016;41(2):65-69.

27. Steinbach $D$, Legrand $O$. ABC transporters and drug resistance in leukemia: was P-gp nothing but the first head of the Hydra?. Leukemia. 2007;21:1172-6.
28. Lei J, Li Q, Gao Y, Zhao L, Liu Y. Increased $\mathrm{PKC} \alpha$ activity by Rak1 overexpression is responsible for chemotherapy resistance in T-cell acute lymphoblastic leukemia-derived cell line. Sci Rep. 2016;6:33717.

29. Hartsink-Segers SA, Zwaan CM, Exalto C, Luijendijk MWJ, Calvert VS, Petricoin EF, et al. Aurora kinases in childhood acute leukemia: the promise of aurora B as therapeutic target. Leukemia. 2013;27(3):560-568.

30. Renner AG, Santos CD, Recher C, Bailly C, Creancier L, Kruczynski A, et al. Polo-like kinase1 is overexpressed in acute myeloid leukemia and its inhibition preferentially targets the proliferation of leukemic cells. Blood. 2009;114(3):659-62.

31. Yakimchuk K, Iravani M, Hasni MS, Rhönnstad P, Nilsson S, Jondal M, et al. Effect of ligand-activated estrogen receptor $\beta$ on lymphoma growth in vitro and in vivo. Leukemia. 2011;25(7):1103-10. 